\title{
Traveling wave solutions in a Lotka-Volterra type competition recursion
}

Shuxia Pan $^{1 *}$ and Pan Yang ${ }^{2}$

${ }^{\text {*Correspondence: shxpan@yeah.net }}$ 1 Department of Applied Mathematics, Lanzhou University of Technology, Lanzhou, Gansu 730050, People's Republic of China Full list of author information is available at the end of the article

\begin{abstract}
This paper is concerned with the traveling wave solutions of a competitive integrodifference system with Lotka-Volterra type nonlinearity. The existence of traveling wave solutions is proved by constructing generalized upper and lower solutions. The asymptotic behavior of traveling wave solutions is established by combining the theory of asymptotic spreading with the idea of contracting rectangles. The nonexistence of monotone traveling wave solutions is also confirmed by the theory of asymptotic spreading.
\end{abstract}

Keywords: competitive interaction; spreading speed; invasion and coexistence

\section{Introduction}

In this paper, we study the traveling wave solutions of the following integrodifference system with Lotka-Volterra type nonlinearity:

$$
\left\{\begin{array}{l}
X_{n+1}(x)=\int_{\mathbb{R}} r_{1} X_{n}(y)\left[1-X_{n}(y)-a_{1} Y_{n}(y)\right] g_{1}(x-y) d y, \\
Y_{n+1}(x)=\int_{\mathbb{R}} r_{2} Y_{n}(y)\left[1-Y_{n}(y)-a_{2} X_{n}(y)\right] g_{2}(x-y) d y,
\end{array}\right.
$$

in which $n \in \mathbb{N} \cup\{0\}$, and $r_{i}>0, a_{i} \geq 0, i=1,2$, are constants. For $i=1,2, g_{i}$ is the probability function describing the random walk of individuals under consideration, and it is the so-called kernel function. In particular, we take the following conditions in this paper:

(A1) $g_{i}$ is Lebesgue measurable and integrable on $\mathbb{R}$ and $\int_{\mathbb{R}} g_{i}(y) d y=1$;

(A2) $g_{i}(y)=g_{i}(-y) \geq 0, y \in \mathbb{R}$, and for each $\lambda \in \mathbb{R}, \int_{\mathbb{R}} g_{i}(y) e^{\lambda y} d y<\infty$.

For the parameters in (1.1), we also give the following assumptions:

(A3) $r_{1}, r_{2} \in(1,4)$;

(A4) $1-l_{1}-a_{1} l_{2} \geq 0,1-l_{2}-a_{2} l_{1} \geq 0$, where

$$
l_{i}= \begin{cases}\frac{r_{i}-1}{r_{i}}, & r_{i} \in(1,2), \\ \frac{r_{i}}{4}, & r_{i} \in[2,4)\end{cases}
$$

for $i=1,2$;

(A5) $r_{1}\left(1-a_{1} l_{2}\right)>1, r_{2}\left(1-a_{2} l_{1}\right)>1$;

(A6) $1-1 / r_{1}>a_{1}\left(1-1 / r_{2}\right), 1-1 / r_{2}>a_{2}\left(1-1 / r_{1}\right)$;

(A7) $1-2 l_{1}-a_{1} l_{2} \geq 0,1-2 l_{2}-a_{2} l_{1} \geq 0$. 
We now give some illustration of (A3)-(A7) from the viewpoint of both the literature and the population dynamics of the corresponding difference system

$$
X_{n+1}=r_{1} X_{n}\left[1-X_{n}-a_{1} Y_{n}\right], \quad Y_{n+1}=r_{2} Y_{n}\left[1-Y_{n}-a_{2} X_{n}\right] .
$$

If the inter-specific vanishes $\left(a_{1}=a_{2}=0\right)$ and (A3) holds in (1.2), then $X_{n+1}=r_{1} X_{n}[1-$ $\left.X_{n}\right]$ or $Y_{n+1}=r_{2} Y_{n}\left[1-Y_{n}\right]:[0,1] \rightarrow[0,1]$ and is persistent in population dynamics (see Murray [1, Section 2.3] for the dynamics). Condition (A4) provides a positive invariant region of the difference system (1.2), namely, $\left[0, l_{1}\right] \times\left[0, l_{2}\right]$. (A5) indicates that the interspecific is weak, namely, even if the competitor $X_{n}\left(Y_{n}\right)$ takes the maximal value $l_{1}\left(l_{2}\right)$, the species $Y_{n}\left(X_{n}\right)$ still persists. The coexistence steady state of (1.2) exists if (A6) holds. (A7) leads to a comparison principle appealing to the difference system (1.2) in $\left[0, l_{1}\right] \times$ $\left[0, l_{2}\right]$. Finally, if (A3)-(A7) hold, then (1.2) has a stable steady state. In particular, there are different parameters such that part or all of (A3)-(A7) hold. For example, if $r_{1}=1.5$, $r_{2}=1.6, a_{1}=a_{2}=0.1$, then (1.2) satisfies (A3)-(A7); if $r_{1}=1.5, r_{2}=1.8, a_{1}=a_{2}=0.5$, then (1.2) satisfies (A3)-(A6), if $r_{1}=1.2, r_{2}=1.8, a_{1}=a_{2}=0.5$, then (A3) and (A4) hold.

Hereafter, a traveling wave solution of (1.1) is a special solution having the form

$$
X_{n}(x)=\phi(t), \quad Y_{n}(x)=\psi(t), \quad t=x+c n,
$$

in which $c>0$ is the wave speed and $(\phi, \psi)$ describes the wave profile that propagates in the media $\mathbb{R}$. Then $(\phi, \psi)$ and $c$ must satisfy

$$
\left\{\begin{array}{l}
\phi(t+c)=\int_{\mathbb{R}} r_{1} \phi(y)\left(1-\phi(y)-a_{1} \psi(y)\right) g_{1}(t-y) d y, \\
\psi(t+c)=\int_{\mathbb{R}} r_{2} \psi(y)\left(1-\psi(y)-a_{2} \phi(y)\right) g_{2}(t-y) d y
\end{array}\right.
$$

for $t \in \mathbb{R}$. To describe determinative transition processes between different states, proper asymptotic behavior satisfied by the traveling wave solutions is often necessary. In this paper, we require the following asymptotic boundary conditions:

$$
\lim _{t \rightarrow-\infty}(\phi(t), \psi(t))=(0,0), \quad \liminf _{t \rightarrow \infty} \phi(t)>0, \quad \liminf _{t \rightarrow \infty} \psi(t)>0 .
$$

Furthermore, we also consider the following stronger ones:

$$
\lim _{t \rightarrow-\infty}(\phi(t), \psi(t))=(0,0), \quad \lim _{t \rightarrow \infty} \phi(t)=k_{1}, \quad \lim _{t \rightarrow \infty} \psi(t)=k_{2},
$$

in which

$$
k_{1}=\frac{1-\frac{1}{r_{1}}-a_{1}\left(1-\frac{1}{r_{2}}\right)}{1-a_{1} a_{2}}>0, \quad k_{2}=\frac{1-\frac{1}{r_{2}}-a_{2}\left(1-\frac{1}{r_{1}}\right)}{1-a_{1} a_{2}}>0,
$$

provided that (A6) is true. In population dynamics, (1.3) with (1.4) or (1.3) with (1.5) could formulate the successful invasion of two competitors.

To study the existence of traveling wave solutions of competitive recursions of two competitors, Lin et al. [2] established an abstract scheme and the existence of traveling wave solutions was reduced to the existence of upper and lower solutions. Since the competitive system in [2] does not generate monotone semiflows when the synchronous invasion of two competitors is concerned, the asymptotic behavior of traveling wave solutions cannot 
be confirmed by the monotonicity of them (see [3-15] for monostable traveling wave solutions of (local) monotone recursions). In [2], the asymptotic behavior of traveling wave solutions was obtained by that of upper and lower solutions. The method was also applied to several competitive systems; see [16-18] and the references cited therein. Without the requirements of upper and lower solutions, it is difficult to obtain the asymptotic behavior of traveling wave solutions [19]. In fact, for coupled systems with general kernels, it is not an easy job to construct proper upper and lower solutions satisfying the asymptotic behavior in [2], and [16-18] just obtained the existence of traveling wave solutions of some systems with special kernels. Although the kernel functions in [2,16-18] satisfy some special conditions, the verification of upper and lower solutions is still very complex, and the nonexistence of nontrivial traveling wave solutions of models in $[2,16-18]$ remains open.

To simplify the construction of upper and lower solutions and provide a more general result of the existence of traveling wave solutions of recursions, Lin [20] further considered the traveling wave solutions of recursions and gave some simpler conditions. By the theory in [20], the existence of traveling wave solutions can be obtained by the existence of upper and lower solutions which are easy to construct. Moreover, by the properties of the corresponding difference systems, the asymptotic behavior of traveling wave solutions was also studied. Moreover, for the model investigated by [2,17], Lin [20] also obtained the nonexistence of nontrivial traveling wave solutions by the theory of asymptotic spreading. In this paper, we shall establish the existence and nonexistence of (1.3) with (1.4) or (1.3) with (1.5), and we present the corresponding mathematical results by the idea in [20]. In particular, we shall not take special general kernels $g_{1}, g_{2}$, and just add conditions (A1)-(A2) in what follows.

The rest of this paper is organized as follows. In Section 2, we investigate the existence of traveling wave solutions by constructing upper and lower solutions and applying Schauder's fixed point theorem in a functional space equipped with the decay norm. In Section 3, the asymptotic boundary conditions (1.4) and (1.5) will be considered by combining the theory of asymptotic spreading with the idea of contracting rectangles in [20]. Finally, the nonexistence of monotone traveling wave solutions is proved, which indicates that the threshold in the paper is the minimal wave speed of monotone traveling wave solutions of (1.1).

\section{Existence of traveling wave solutions}

In this paper, we shall utilize standard partial ordering in $\mathbb{R}^{2}$. Also let $X$ be the set of uniformly continuous and bounded functions from $\mathbb{R}$ to $\mathbb{R}^{2}$. Moreover, we denote

$$
X_{[\mathbf{0 , \mathbf { L }}]}=\left\{\Phi=(\phi, \psi) \in X: 0 \leq \psi(t) \leq l_{1}, 0 \leq \phi(t) \leq l_{2}, t \in \mathbb{R}\right\} .
$$

Let $|\cdot|$ be the supremum norm in $\mathbb{R}^{2}$ and $\mu>0$ be a constant. We define

$$
B_{\mu}\left(\mathbb{R}, \mathbb{R}^{2}\right)=\left\{\Phi \in X: \sup _{x \in \mathbb{R}}|\Phi(x)| e^{-\mu|x|}<\infty\right\},
$$

and the decay norm

$$
|\Phi|_{\mu}=\sup _{x \in \mathbb{R}}|\Phi(x)| e^{-\mu|x|}, \quad \Phi \in B_{\mu}\left(\mathbb{R}, \mathbb{R}^{2}\right) .
$$

Then $\left(B_{\mu}\left(\mathbb{R}, \mathbb{R}^{2}\right),|\cdot|_{\mu}\right)$ is a Banach space. 
Before discussing (1.1), we first consider the corresponding difference equation

$$
\begin{cases}X_{n+1}=r_{1} X_{n}\left[1-X_{n}-a_{1} Y_{n}\right], & X_{0}=x_{0}, \\ Y_{n+1}=r_{2} Y_{n}\left[1-Y_{n}-a_{2} X_{n}\right], & Y_{0}=y_{0}, \quad n+1 \in \mathbb{N} .\end{cases}
$$

Lemma 2.1 For (2.1), we have the following conclusions:

(D1) if (A3)-(A4) hold, then $\left[0, l_{1}\right] \times\left[0, l_{2}\right]$ is invariant, namely, if $x_{0} \in\left[0, l_{1}\right], y_{0} \in\left[0, l_{2}\right]$, then $X_{n} \in\left[0, l_{1}\right], Y_{n} \in\left[0, l_{2}\right], n \in \mathbb{N}$;

(D2) if (A3), (A4), and (A7) are true and $x_{0} \in\left[0, l_{1}\right], y_{0} \in\left[0, l_{2}\right]$, then for each $n \in \mathbb{N}$, $X_{n}\left(Y_{n}\right)$ is monotone increasing in $X_{n-1}\left(Y_{n-1}\right)$ and monotone decreasing in $Y_{n-1}$ $\left(X_{n-1}\right)$.

We now introduce some constants. Denote

$$
\Lambda_{i}(\lambda, c)=r_{i} \int_{\mathbb{R}} e^{\lambda y-\lambda c} g_{i}(y) d y, \quad i=1,2
$$

for $\lambda \in \mathbb{R}$ and $c \geq 0$. Then the following result holds.

Lemma 2.2 There exists a positive constant $c^{*}>0$ such that $c<c^{*}$ implies that $\Lambda_{1}(\lambda, c)>1$ for any $\lambda \geq 0$ or $\Lambda_{2}(\lambda, c)>1$ for any $\lambda \geq 0$. If $c>c^{*}$, then $\Lambda_{i}(\lambda, c)=1$ has at least one positive root $\lambda_{i}(c)$ such that $\Lambda_{i}\left(\lambda_{i}(c), c\right)=1$ and $\Lambda_{i}(\lambda, c)>1$ for $\lambda \in\left(0, \lambda_{i}(c)\right), i=1,2$. Moreover, there exists $\gamma=\gamma(c) \in(1,2)$ such that $\Lambda_{i}\left(\lambda_{i}^{\prime}(c), c\right)<1$ for all $\lambda_{i}^{\prime}(c) \in\left(\lambda_{i}(c), \gamma \lambda_{i}(c)\right], i=1,2$.

By Liang and Zhao [21, Lemma 3.8], Lemma 2.2 is clear and $c^{*}$ is defined by

$$
c^{*}=\max \left\{\inf _{\lambda>0} \frac{\ln \left(r_{1} \int_{\mathbb{R}} e^{\lambda y} g_{1}(y) d y\right)}{\lambda}, \inf _{\lambda>0} \frac{\ln \left(r_{2} \int_{\mathbb{R}} e^{\lambda y} g_{2}(y) d y\right)}{\lambda}\right\} .
$$

In what follows, we suppose that $c>c^{*}$ is fixed. If $(\phi, \psi) \in X_{[0, \mathbf{L}]}$, then we define $P=$ $\left(P_{1}, P_{2}\right): X_{[0, \mathbf{L}]} \rightarrow X$ by

$$
\begin{aligned}
& P_{1}(\phi, \psi)(t)=\int_{\mathbb{R}} r_{1} \phi(y)\left(1-\phi(y)-a_{1} \psi(y)\right) g_{1}(t-c-y) d y, \\
& P_{2}(\phi, \psi)(t)=\int_{\mathbb{R}} r_{2} \psi(y)\left(1-\psi(y)-a_{2} \phi(y)\right) g_{2}(t-c-y) d y .
\end{aligned}
$$

In particular, $P$ also admits the following properties.

Lemma 2.3 $P: X_{[0, \mathbf{L}]} \rightarrow X_{[0, \mathbf{L}]}$.

Lemma 2.3 is clear by Lemma 2.1 and (A1)-(A2), and we omit the proof here. Clearly, a fixed point of $P$ in $X_{[0, \mathbf{L}]}$ is a solution to (1.3). Therefore, it suffices to prove the existence of the fixed points of $P$ by Schauder's fixed point theorem, and we first construct a potential set of wave profiles.

For the purpose, we define continuous functions

$$
\bar{\phi}(t)=\min \left\{e^{\lambda_{1} t}, l_{1}\right\}, \quad \bar{\psi}(t)=\min \left\{e^{\lambda_{2} t}, l_{2}\right\}
$$


and

$$
\underline{\phi}(t)=\max \left\{e^{\lambda_{1} t}-M e^{\eta \lambda_{1} t}, 0\right\}, \quad \underline{\psi}(t)=\max \left\{e^{\lambda_{2} t}-M e^{\eta \lambda_{2} t}, 0\right\},
$$

where $M>1$ is a positive constant clarified later and $\eta \in(1,2)$ such that

$$
\eta \lambda_{1}<\lambda_{1}+\lambda_{2}, \quad \eta \lambda_{2}<\lambda_{1}+\lambda_{2}, \quad \Lambda_{1}\left(\eta \lambda_{1}, c\right)<1, \quad \Lambda_{2}\left(\eta \lambda_{2}, c\right)<1 .
$$

With these notations, we define the following set of potential wave profiles:

$$
\Gamma=\{(\phi, \psi) \in X,(\underline{\phi}, \underline{\psi}) \leq(\phi, \psi) \leq(\bar{\phi}, \bar{\psi})\},
$$

which satisfies the following nice properties.

Lemma 2.4 If $M>1$ is large such that

$$
(\underline{\phi}, \underline{\psi}) \leq(\bar{\phi}, \bar{\psi})
$$

then $\Gamma$ is convex and nonempty. Moreover, it is closed and bounded with respect to the decay norm $|\cdot|_{\mu}$.

Lemma 2.5 Assume that (A3)-(A4) hold. If $M>1$ is large, then $P: \Gamma \rightarrow \Gamma$.

Proof By Lemma 2.1, it suffices to verify that

$$
\left(P_{1}(\phi, \psi)(t), P_{2}(\phi, \psi)(t)\right) \leq(\bar{\phi}(t), \bar{\psi}(t)), \quad t \in \mathbb{R}
$$

and

$$
\left(P_{1}(\phi, \psi)(t), P_{2}(\phi, \psi)(t)\right) \geq(\underline{\phi}(t), \underline{\psi}(t)), \quad t \in \mathbb{R}
$$

for $(\phi, \psi) \in \Gamma$.

If $\bar{\phi}(t)=e^{\lambda_{1} t}$, then

$$
\begin{aligned}
P_{1}(\phi, \psi)(t) & =\int_{\mathbb{R}} r_{1} \phi(y)\left(1-\phi(y)-a_{1} \psi(y)\right) g_{1}(t-c-y) d y \\
& \leq \int_{\mathbb{R}} r_{1} \phi(y) g_{1}(t-c-y) d y \\
& \leq \int_{\mathbb{R}} r_{1} e^{\lambda_{1} y} g_{1}(t-c-y) d y \\
& =e^{\lambda_{1} t}=\bar{\phi}(t) .
\end{aligned}
$$

If $\bar{\phi}(t)=l_{1}$, then Lemma 2.1 implies that

$$
\begin{aligned}
P_{1}(\phi, \psi)(t) & =\int_{\mathbb{R}} r_{1} \phi(y)\left(1-\phi(y)-a_{1} \psi(y)\right) g_{1}(t-c-y) d y \\
& \leq \int_{\mathbb{R}} r_{1} \phi(y)(1-\phi(y)) g_{1}(t-c-y) d y \\
& \leq l_{1}=\bar{\phi}(t),
\end{aligned}
$$


and we have verified that

$P_{1}(\phi, \psi)(t) \leq \bar{\phi}(t), \quad t \in \mathbb{R}$.

In a similar way, we can prove that

$P_{2}(\phi, \psi)(t) \leq \bar{\psi}(t), \quad t \in \mathbb{R}$.

If $\phi(t)=0$, then

$$
\begin{aligned}
1-\phi(y)-a_{1} \psi(y) & \geq 1-\bar{\phi}(y)-a_{1} \bar{\psi}(y) \\
& \geq 1-l_{1}-a_{1} l_{2} \\
& \geq 0=\underline{\phi}(y)
\end{aligned}
$$

by (A4) and

$$
P_{1}(\phi, \psi)(t)=\int_{\mathbb{R}} r_{1} \phi(y)\left(1-\phi(y)-a_{1} \psi(y)\right) g_{1}(t-c-y) d y \geq 0=\underline{\phi}(t)
$$

is clear. Otherwise, $(\phi, \psi) \in \Gamma$ leads to

$$
\begin{aligned}
r_{1} \phi(y)\left(1-\phi(y)-a_{1} \psi(y)\right) & =r_{1} \phi(y)-r_{1} \phi^{2}(y)-r_{1} a_{1} \phi(y) \psi(y) \\
& \geq r_{1} e^{\lambda_{1} y}-r_{1} M e^{\eta \lambda_{1} y}-r_{1} e^{2 \lambda_{1} y}-r_{1} a_{1} e^{\left(\lambda_{1}+\lambda_{2}\right) y}
\end{aligned}
$$

and

$$
\begin{aligned}
P_{1}(\phi, \psi)(t)= & \int_{\mathbb{R}} r_{1} \phi(y)\left(1-\phi(y)-a_{1} \psi(y)\right) g_{1}(t-c-y) d y \\
\geq & \int_{\mathbb{R}}\left[r_{1} e^{\lambda_{1} y}-r_{1} M e^{\eta \lambda_{1} y}\right] g_{1}(t-c-y) d y \\
& -\int_{\mathbb{R}}\left[r_{1} e^{2 \lambda_{1} y}+r_{1} a_{1} e^{\left(\lambda_{1}+\lambda_{2}\right) y}\right] g_{1}(t-c-y) d y \\
= & e^{\lambda_{1} t}-M \Lambda_{1}\left(\eta \lambda_{1}, c\right) e^{\eta \lambda_{1} t}-\Lambda_{1}\left(2 \lambda_{1}, c\right) e^{2 \lambda_{1} t}-a_{1} \Lambda_{1}\left(\lambda_{1}+\lambda_{2}, c\right) e^{\left(\lambda_{1}+\lambda_{2}\right) t} \\
\geq & e^{\lambda_{1} t}-M e^{\eta \lambda_{1} t} \\
= & \underline{\phi}(t)
\end{aligned}
$$

provided that

$$
M \geq 1+\frac{\Lambda_{1}\left(2 \lambda_{1}, c\right)+a_{1} \Lambda_{1}\left(\lambda_{1}+\lambda_{2}, c\right)}{1-\Lambda_{1}\left(\eta \lambda_{1}, c\right)}>1 .
$$

In a similar way, when

$$
M \geq 1+\frac{\Lambda_{2}\left(2 \lambda_{2}, c\right)+a_{2} \Lambda_{2}\left(\lambda_{1}+\lambda_{2}, c\right)}{1-\Lambda_{2}\left(\eta \lambda_{1}, c\right)}>1
$$


is true, we have

$$
P_{2}(\phi, \psi)(t) \geq \underline{\psi}(t), \quad t \in \mathbb{R} .
$$

The proof is complete.

Lemma 2.6 Assume that (A3)-(A4) hold. Then $P: \Gamma \rightarrow \Gamma$ is complete continuous in the sense of the decay norm $|\cdot|_{\mu}$.

The proof is provided by Lin [20, Lemma 3.4] and we omit it here.

Theorem 2.7 Assume that (A3)-(A4) hold. Then for each $c>c^{*}$, (1.3) has a positive solution $(\phi, \psi)$ such that

$$
\lim _{t \rightarrow-\infty}(\phi(t), \psi(t))=(0,0), \quad \lim _{t \rightarrow-\infty}\left(\phi(t) e^{-\lambda_{1} t}, \psi(t) e^{-\lambda_{2} t}\right)=(1,1)
$$

and

$$
0<\phi(t) \leq l_{1}, \quad 0<\psi(t) \leq l_{2}, \quad t \in \mathbb{R} .
$$

The result is evident by Schauder's fixed point theorem and Lemmas 2.4-2.6, and we omit the proof here.

\section{Asymptotic behavior of traveling wave solutions}

In this section, we investigate the asymptotic behavior of positive solutions of (1.3). We first consider the initial value problem

$$
\left\{\begin{array}{l}
u_{n+1}(x)=\int_{\mathbb{R}} b\left(u_{n}(y)\right) g(x-y) d y, \\
u_{0}(x)=u(x), \quad x \in \mathbb{R}, n+1 \in \mathbb{N},
\end{array}\right.
$$

where $b(u)$ satisfies

(U1) for some $U^{+}>0, b(u):\left[0, U^{+}\right] \rightarrow\left[0, U^{+}\right]$is Lipschitz continuous and monotone increasing;

(U2) there exists $u^{+} \in\left(0, U^{+}\right]$such that $b(u)>u, u \in\left(0, u^{+}\right), b\left(u^{+}\right)=u^{+}$; if $U^{+}>u^{+}$, then $b(u)<u, u \in\left(u^{+}, U^{+}\right)$

(U3) $b(0)=0$;

(U4) $b^{\prime}(0)>1$ and $b(u)<b^{\prime}(0) u, u \in\left(0, U^{+}\right]$.

In literature, (U1)-(U4) imply the comparison principle, monostability, and persistence in (3.1). More precisely, by Hsu and Zhao [4], we have the following conclusion.

Lemma 3.1 Assume that $g$ satisfies (A1)-(A2).

(1) If $u(x)$ is bounded and uniformly continuous such that

$$
0<u(x) \leq U^{+}, \quad x \in \mathbb{R},
$$


then $\lim _{n \rightarrow \infty} u_{n}(0)=u^{+}$. Moreover, let $C>0$ satisfy

$$
\left\{\begin{array}{l}
\int_{\mathbb{R}} b^{\prime}(0) e^{\lambda y-\lambda c} g(y) d y=1 \text { has no real root } \lambda \geq 0 \text { if } c<C, \\
\int_{\mathbb{R}} b^{\prime}(0) e^{\lambda y-\lambda c} g(y) d y=1 \text { has positive roots if } c \geq C,
\end{array}\right.
$$

then for each $c<C$, we have

$$
\liminf _{n \rightarrow \infty} \inf _{|x|<c n} u_{n}(x)=\limsup _{n \rightarrow \infty} \sup _{|x|<c n} u_{n}(x)=u^{+} .
$$

(2) If $\bar{u}_{n}(x)$ is bounded and uniformly continuous for each $n$ such that

$$
0 \leq \bar{u}_{n}(x) \leq U^{+}, \quad n+1 \in \mathbb{N}, x \in \mathbb{R}
$$

and

$$
\left\{\begin{array}{l}
\bar{u}_{n+1}(x) \geq(\leq) \int_{\mathbb{R}} b\left(\bar{u}_{n}(y)\right) g(x-y) d y \\
\bar{u}_{0}(x) \geq(\leq) u(x), \quad x \in \mathbb{R}, n+1 \in \mathbb{N}
\end{array}\right.
$$

then $\bar{u}_{n}(x) \geq(\leq) u_{n}(x), x \in \mathbb{R}, n \in \mathbb{N}$.

Theorem 3.2 Assume that (A3)-(A5) hold. If $(\phi, \psi)$ is given by Theorem 2.7, then (1.4) is true.

Proof Note that a traveling wave solution is a special invariant solution; then $\phi(t)=X_{n}(x)$ satisfies the following inequality:

$$
\left\{\begin{array}{l}
X_{n+1}(x) \geq \int_{\mathbb{R}} r_{1} X_{n}(y)\left[1-X_{n}(y)-a_{1} l_{2}\right] g_{1}(x-y) d y \\
X_{0}(x)=\phi(x) \in\left[0, l_{1}\right]
\end{array}\right.
$$

for all $n+1 \in \mathbb{N}$. Define

$$
\underline{b}(u)=\inf _{v \in\left[u, l_{1}\right]}\left\{r_{1} v\left[1-v-a_{1} l_{2}\right]\right\} .
$$

Clearly, $\underline{b}(u)$ admits the following properties:

(b1) $\underline{b}(u):\left[0, l_{1}\right] \rightarrow\left[0, l_{1}\right]$ is Lipschitz continuous and monotone increasing;

(b2) $\underline{b}(0)=0, \underline{b}^{\prime}(0)=r_{1}\left(1-a_{1} l_{2}\right)>1$;

(b3) there exists $\underline{u}^{+} \in\left(0, l_{1}\right]$ such that $\underline{b}\left(\underline{u}^{+}\right)=\underline{u}^{+}$and

$$
\underline{b}(u)>u, \quad u \in\left(0, \underline{u}^{+}\right)
$$

(b4) if $\underline{u}^{+}<l_{1}$, then $\underline{b}(u)<u, u \in\left(\underline{u}^{+}, l_{1}\right]$

Therefore, $\underline{b}(u)$ satisfies (U1)-(U4) and Lemma 3.1 implies that

$$
\liminf _{n \rightarrow \infty} X_{n}(0) \geq \underline{u}^{+}>0
$$


and

$$
\frac{r_{1}}{4} \geq \liminf _{t \rightarrow \infty} \phi(t) \geq \underline{u}^{+}>0 .
$$

In a similar way, we have

$$
\frac{r_{2}}{4} \geq \liminf _{t \rightarrow \infty} \psi(t)>0
$$

The proof is complete.

Theorem 3.3 Assume that (A3)-(A7) hold. If $(\phi, \psi)$ is given by Theorem 2.7, then (1.5) is true.

Proof By what we have done, we see that

$$
\frac{r_{1}}{4} \geq \limsup _{t \rightarrow \infty} \phi(t) \geq \liminf _{t \rightarrow \infty} \phi(t)>0
$$

and

$$
\frac{r_{2}}{4} \geq \limsup _{t \rightarrow \infty} \psi(t) \geq \liminf _{t \rightarrow \infty} \psi(t)>0 .
$$

Define

$$
\liminf _{t \rightarrow \infty} \phi(t)=\underline{u}, \quad \quad \limsup _{t \rightarrow \infty} \phi(t)=\bar{u}
$$

and

$$
\liminf _{t \rightarrow \infty} \psi(t)=\underline{v}, \quad \limsup _{t \rightarrow \infty} \psi(t)=\bar{v} .
$$

Applying the dominated convergence theorem in $P$ when $t \rightarrow \infty$, then the monotone condition (A7) implies that

$$
\begin{aligned}
& \bar{u}+a_{1} \underline{v} \leq 1-\frac{1}{r_{1}}, \\
& \underline{v}+a_{2} \bar{u} \geq 1-\frac{1}{r_{2}}, \\
& \underline{u}+a_{1} \bar{v} \geq 1-\frac{1}{r_{1}}, \\
& \bar{v}+a_{2} \underline{u} \leq 1-\frac{1}{r_{2}} .
\end{aligned}
$$

By (3.2) and (3.4), we have

$$
\bar{u}-\underline{u} \leq a_{1}(\bar{v}-\underline{v}) .
$$

Furthermore, (3.3) and (3.5) lead to

$$
\bar{v}-\underline{v} \leq a_{2}(\bar{u}-\underline{u}) .
$$


Since (A6) implies that $a_{1} a_{2}<1$, then we have

$$
\bar{u}=\underline{u}, \quad \bar{v}=\underline{v}
$$

by (3.6) and (3.7). Again by (A6), we see that

$$
\bar{u}=\underline{u}=k_{1}, \quad \bar{v}=\underline{v}=k_{2} .
$$

The proof is complete.

Remark 3.4 Although we did not construct the contracting rectangle (see [20]) in this paper, the proof of Theorem 3.3 was motivated by Lin [20, Sections 4-5]. Of course, if a model involves more unknown functions, it is difficult to obtain the asymptotic behavior of traveling wave solutions by the inequalities similar to (3.6) and (3.7).

\section{Nonexistence of monotone traveling wave solutions}

In this section, we confirm that $c^{*}$ is the minimal wave speed of monotone invasion traveling wave solutions by presenting the following nonexistence of monotone traveling wave solutions.

Theorem 4.1 Assume that (A3)-(A6) hold. If $c<c^{*}$, then there is no strict monotone $(\phi(t), \psi(t))$ satisfying (1.3)-(1.4) and

$$
0<\phi(t)<k_{1}, \quad 0<\psi(t)<k_{2} .
$$

Proof Without loss of generality, we assume that $a_{1}>0$ and

$$
\Lambda_{1}(\lambda, c)>1, \quad c \in\left(0, c^{*}\right), \lambda \geq 0 .
$$

Were the statement false, then there exists $c_{1} \in\left(0, c^{*}\right)$ such that there is $(\phi(t), \psi(t))$ satisfying (1.3)-(1.4) and (4.1).

Since $\phi(t), \psi(t)$ are monotone increasing, then

$$
t \rightarrow \phi(t), \quad t \rightarrow \psi(t)
$$

are one to one and there is a continuous mapping $h$ such that

$$
h(\phi(t))=r_{1}\left(1-\phi(t)-a_{1} \psi(t)\right),
$$

in which $h:\left[0, k_{1}\right] \rightarrow\left[1, r_{1}\right]$ satisfying

(H1) $h(0)=r_{1}$

$(\mathrm{H} 2) h:\left[0, k_{1}\right] \rightarrow\left[1, r_{1}\right]$ is continuous and strictly monotone decreasing;

(H3) $h\left(k_{1}\right)=1$.

At the same time, $h$ also leads to

$$
\phi\left(t+c_{1}\right)=\int_{\mathbb{R}} \phi(y) h(\phi(y)) g_{1}(t-y) d y, \quad t \in \mathbb{R} .
$$


Let $-2 x=\left(c_{1}+c^{*}\right) n$, then

$$
t=x+c_{1} n \rightarrow-\infty, \quad n \rightarrow \infty
$$

such that

$$
\phi(t) \rightarrow 0, \quad t \rightarrow-\infty
$$

Note that $\phi(t)=u_{n}(x)$ is a special solution to

$$
\left\{\begin{array}{l}
u_{n+1}(x)=\int_{\mathbb{R}} h\left(u_{n}(y)\right) g_{1}(x-y) d y, \\
u_{0}(x)=\phi(x)>0, \quad x \in \mathbb{R}, n+1 \in \mathbb{N} .
\end{array}\right.
$$

Define $\underline{h}(u)=\inf _{v \in\left[u, k_{1}\right]} h(v)$, then $h(u)$ is monotone and continuous for $u \in\left[0, k_{1}\right]$. Moreover, applying Lemma 3.1 to (4.2), we see that $C=c^{*}$. Again by Lemma 3.1, we obtain

$$
\liminf _{n \rightarrow \infty,-2 x=\left(c_{1}+c^{*}\right) n} u_{n}(x)>0
$$

and

$$
\liminf _{t \rightarrow-\infty} \phi(t)>0
$$

A contradiction occurs. The proof is complete.

Before ending this paper, we make the following remark.

Remark 4.2 By what we have done, $c^{*}$ is the minimal wave speed of monotone traveling wave solutions of (1.1). However, when the wave speed is $c^{*}$, the existence and nonexistence of nontrivial traveling wave solutions remain open. At the same time, if we remove the monotonicity of traveling wave solutions in Theorem 4.1, then we believe that the result still holds. Clearly, for these two problems, we cannot discuss them directly by the methods similar to those in this paper, and we shall consider these problems in our future studies.

Competing interests

The authors declare that they have no competing interests.

Authors' contributions

All authors contributed equally to the manuscript. All authors read and approved the final manuscript.

\section{Author details}

'Department of Applied Mathematics, Lanzhou University of Technology, Lanzhou, Gansu 730050, People's Republic of China. ${ }^{2}$ School of Mathematics and Statistics, Lanzhou University, Lanzhou, Gansu 730000, People's Republic of China.

\section{Acknowledgements}

The first author was supported by NSF of Gansu Province of China (No. 1208RJYA004) and the Development Program for Outstanding Young Teachers in Lanzhou University of Technology (No. 1010ZCX019), the second author was supported by National Undergraduate Training Programs for Innovation and Entrepreneurship (No. 201310730086). 


\section{References}

1. Murray, JD: Mathematical Biology I: An Introduction. Interdisciplinary Applied Mathematics, vol. 17. Springer, New York (2002)

2. Lin, G, Li, W-T, Ruan, S: Spreading speeds and traveling waves in competitive recursion systems. J. Math. Biol. 62(2), 165-201 (2011)

3. Creegan, P, Lui, R: Some remarks about the wave speed and travelling wave solutions of a nonlinear integral operator. J. Math. Biol. 20(1), 59-68 (1984)

4. Hsu, S-B, Zhao, X-Q: Spreading speeds and traveling waves for nonmonotone integrodifference equations. SIAM J. Math. Anal. 40(2), 776-789 (2008)

5. Kot, M: Discrete-time travelling waves: ecological examples. J. Math. Biol. 30(4), 413-436 (1992)

6. Li, B, Lewis, MA, Weinberger, HF: Existence of traveling waves for integral recursions with nonmonotone growth functions. J. Math. Biol. 58(3), 323-338 (2009)

7. Lin, G, Li, W-T: Spreading speeds and traveling wavefronts for second order integrodifference equations. J. Math. Anal. Appl. 361(2), 520-532 (2010)

8. Lin, G, Li, W-T, Ruan, S: Asymptotic stability of monostable wavefronts in discrete-time integral recursions. Sci. China Math. 53(5), 1185-1194 (2010)

9. Lui, R: Biological growth and spread modeled by systems of recursions. I. Mathematical theory. Math. Biosci. 93(2), 269-295 (1989)

10. Pan, S, Lin, G: Propagation of second order integrodifference equations with local monotonicity. Nonlinear Anal., Real World Appl. 12(1), 535-544 (2011)

11. Wang, H, Castillo-Chavez, C: Spreading speeds and traveling waves for non-cooperative integro-difference systems. Discrete Contin. Dyn. Syst., Ser. B 17(6), 2243-2266 (2012)

12. Weinberger, HF: Long-time behavior of a class of biological models. SIAM J. Math. Anal. 13(3), 353-396 (1982)

13. Weinberger, HF: On spreading speeds and traveling waves for growth and migration models in a periodic habitat. J. Math. Biol. 45(6), 511-548 (2002)

14. Weinberger, HF, Kawasaki, K, Shigesada, N: Spreading speeds of spatially periodic integro-difference models for populations with nonmonotone recruitment functions. J. Math. Biol. 57(3), 387-411 (2008)

15. Weinberger, HF, Lewis, MA, Li, B: Analysis of linear determinacy for spread in cooperative models. J. Math. Biol. 45(3), 183-218 (2002)

16. Li, K, Li, X: Travelling wave solutions in integro-difference competition system. IMA J. Appl. Math. 78(3), 633-650 (2013)

17. Lin, G, Li, W-T: Traveling wave solutions of a competitive recursion. Discrete Contin. Dyn. Syst., Ser. B 17(1), 173-189 (2012)

18. Zhu, F, Lin, G: Propagation of a difference-integral competitive system I: traveling wave solutions. Sciencepaper Online: http://www.paper.edu.cn/releasepaper/content/201204-75 (2012)

19. Li, B: Some remarks on traveling wave solutions in competition models. Discrete Contin. Dyn. Syst., Ser. B 12, 389-399 (2009)

20. Lin, G: Traveling wave solutions for integro-difference systems. arXiv preprint arXiv:1305.4031 (2013)

21. Liang, X, Zhao, X-Q: Asymptotic speeds of spread and traveling waves for monotone semiflows with applications. Commun. Pure Appl. Math. 60(1), 1-40 (2007)

10.1186/1687-1847-2014-173

Cite this article as: Pan and Yang: Traveling wave solutions in a Lotka-Volterra type competition recursion. Advances in Difference Equations 2014, 2014:173

\section{Submit your manuscript to a SpringerOpen ${ }^{\circ}$ journal and benefit from:}

- Convenient online submission

- Rigorous peer review

- Immediate publication on acceptance

- Open access: articles freely available online

- High visibility within the field

- Retaining the copyright to your article 